\title{
Antimicrobial stewardship: Staff nurse knowledge and attitudes
}

Katreena Collette Merrill

Brigham Young University - Provo, katreena.merrill@byu.edu

Sandra Forsyth Hanson

Sharon Sumner

Todd Vento

John Veillette

See next page for additional authors

Follow this and additional works at: https://scholarsarchive.byu.edu/facpub

Part of the Other Nursing Commons

\section{Original Publication Citation}

Merrill, K.C., Hanson, S.F., Sumner, S., Vento, T., Veillette, J., \& Webb, B. (2019). Antimicrobial Stewardship: Staff Nurse Knowledge and Attitudes. American Journal of Infection Control. 47(10), 1219-1224.

\section{BYU ScholarsArchive Citation}

Merrill, Katreena Collette; Hanson, Sandra Forsyth; Sumner, Sharon; Vento, Todd; Veillette, John; and Webb, Brandono, "Antimicrobial stewardship: Staff nurse knowledge and attitudes" (2019). Faculty Publications. 5256.

https://scholarsarchive.byu.edu/facpub/5256

This Peer-Reviewed Article is brought to you for free and open access by BYU ScholarsArchive. It has been accepted for inclusion in Faculty Publications by an authorized administrator of BYU ScholarsArchive. For more information, please contact ellen_amatangelo@byu.edu. 


\section{Authors}

Katreena Collette Merrill, Sandra Forsyth Hanson, Sharon Sumner, Todd Vento, John Veillette, and Brandono Webb 


\title{
Major Article
}

\section{Antimicrobial stewardship: Staff nurse knowledge and attitudes}

\author{
Katreena Merrill PhD, RN ${ }^{\mathrm{a}, *}$, Sandra Forsyth Hanson BS, RN, CIC ${ }^{b}$, Sharon Sumner BS, RN, CIC ${ }^{c}$, \\ Todd Vento MD, $\mathrm{MPH}^{\mathrm{d}}$, John Veillette PharmD ${ }^{\mathrm{e}}$, Brandon Webb MD ${ }^{\mathrm{f}}$ \\ ${ }^{a}$ College of Nursing, Brigham Young University, Provo, UT \\ ${ }^{\mathrm{b}}$ Infection Prevention, LDS Hospital, Salt Lake City, UT \\ ${ }^{\mathrm{C}}$ Infection Prevention, Intermountain Medical Center, Murray, UT \\ ${ }^{\mathrm{d}}$ Infectious Diseases TeleHealth, Division of Infectious Diseases and Epidemiology, Intermountain Medical Center, Murray, UT \\ e Infectious Diseases, Intermountain Medical Center, Murray, UT \\ ${ }^{\mathrm{f}}$ Infectious Diseases, LDS Hospital, Salt Lake City, UT
}

\section{Key Words:}

Knowledge/attitudes

Nursing

Antimicrobial resistance

Allergy

\begin{abstract}
Background: Registered nurses are uniquely qualified to augment antimicrobial stewardship (AS) processes. However, the role of nursing in AS needs further development. More information is needed regarding gaps in registered nurse knowledge, attitudes toward AS, and how infection preventionists can help.

Methods: An online descriptive survey was deployed to a convenience sample of approximately 2,000 nurses at the bedside. The survey included 15 questions addressing: (1) overall knowledge of AS; (2) antimicrobial delivery; (3) knowledge and attitudes regarding antimicrobial use; (4) antimicrobial resistance; and (5) antimicrobial resources and education.

Results: Three hundred sixteen staff nurses from 3 hospitals (15.8\%) responded to the survey. Fifty-two percent of nurses were not familiar with the term "antimicrobial stewardship," although 39.6\% of nurses indicated that an AS program was moderately or extremely important in their health care setting. Almost all nurses (95\%) believed that they should be involved in AS interventions.
\end{abstract}

Discussion: These findings suggest gaps in nursing knowledge rearding AS. However, nurses believed AS programs were important and were eager to be involved.

Conclusions: This study showed that many nurses are not aware of AS, or do not understand their role in contributing to AS endeavors. Infection preventionist education should focus on increasing staff nurse awareness and demonstrating how nurses can make specific AS interventions.

(C) 2019 Association for Professionals in Infection Control and Epidemiology, Inc. Published by Elsevier Inc. All rights reserved.
According to the Centers for Disease Control and Prevention (CDC), ${ }^{1}$ each year, 2 million cases of antimicrobial-resistant bacteria occur resulting in approximately 23,000 deaths. As a result, the CDC and others recommend the implementation of antimicrobial stewardship (AS) programs..$^{2-4}$ AS refers to strategies to limit the use of antimicrobial agents to the minimum necessary spectrum and duration when antimicrobials are indicated. The goal of AS is to reduce the development of antimicrobial resistance and to improve patient outcomes. AS affects all venues of patient care, including outpatient, inpatient, and long-term care. Although many strategies for AS exist

\footnotetext{
* Address correspondence to Katreena Merrill, PhD, RN, Brigham Young University College of Nursing, 500 Spencer Kimball Tower, Provo, UT 84602.

E-mail address: katreena.merrill@byu.edu (K. Merrill).

Funding/support: This work was supported by internal funding from Brigham Young University College of Nursing.

Conflicts of interest: None to report.
}

and best-practices continue to evolve, successful AS programs use a multidisciplinary approach that takes place at the point of care. ${ }^{4}$

In 2017, the American Nurses Association and the CDC issued a joint white paper outlining the role of the registered nurse (RN) in the hospital AS practices. ${ }^{2}$ The American Nurses Association and CDC, along with many others, openly support the premise that RNs are uniquely qualified to augment AS and play an essential role in the AS process. ${ }^{4-7}$ Although there are some efforts to engage nurses in AS, more strategies are needed to explore the role of nursing in $\mathrm{AS}^{8}$

The role of the infection preventionist (IP) is to prevent health care-acquired infections. IPs have a strong relationship with nursing and other disciplines. Because IPs exhibit more extensive knowledge of microbiology and resistance, this makes them an ideal AS nurse educator and a valuable interface between nursing and other AS leaders. $^{3}$ As IPs already function in this capacity, participation in nursing AS education is a natural extension. 
More information is needed regarding gaps in RN knowledge, attitudes toward AS, and how IPs can help bridge those gaps. Therefore, the purpose of this study is to describe the nurse's self-reported knowledge of antimicrobial use and resistance and gauge their attitude toward involvement in AS efforts.

\section{METHODS}

The study was a descriptive survey design. A convenience sample of approximately 2,000 nurses at the bedside from 3 hospitals in an integrated health system in Utah were sent an e-mail link to participate in the study. The participating hospitals had approximately 40 , 250 , and 500 inpatient beds, respectively. Each hospital was in varying degrees of implementing their AS program; however, at the time of the survey, none of the AS teams included nurses at the point of care.

After review and approval by the institutional review board, a recruitment e-mail including a short description of the study and a link to an electronic questionnaire using Qualtrics (www.qualtrics. com) was sent to participants by their nurse administrator. A second follow-up e-mail was sent 2 weeks later to increase the response rate. Those who completed the survey were entered into a random drawing for a gift card. A total of 100 gift cards were distributed.

\section{Instruments}

At the time of the study design, there were no existing valid and reliable instruments to measure AS in nurses. A descriptive questionnaire, modified from previous studies, was explicitly designed for nurses by the research team. The questionnaire was reviewed for face validity by a multidisciplinary team of 5 AS committee members from the study hospitals. The questionnaire was updated based on their feedback and prepared for online distribution. The questionnaire took approximately 15 minutes to complete.

The questionnaire included 15 questions (with sub-questions resulting in 27 items) and 8 demographic questions (experience, sex, age, ethnicity, education, role, specialty, and hospital). The questionnaire assessed 5 components of AS: (1) overall knowledge about AS; (2) delivery of antimicrobials; (3) knowledge and attitudes about antimicrobial use; (4) knowledge and attitudes about antimicrobial resistance, and (5) resources and education about antimicrobials. The full questionnaire is provided in supplemental materials and is described briefly in the following text (Supplementary Fig S1).

Data were reviewed for completeness and analyzed using SPSS version 24 (IBM Corp, Armonk, NY) for analysis. Descriptive statistics (mean, SD, skew, and kurtosis) were performed, and each variable was assessed for normality of distribution. Percentages were calculated for categorical variables. Mean scores were calculated for the Likert-type scales. Cronbach's alpha measured the internal consistency of the instrument. A 1-way analysis of variance was used to determine if there was a difference in mean responses and the $\chi^{2}$ test was used to assess differences in categorical variables. The Pearson correlation statistic was completed to detect the relationship between knowledge, age, and experience. A $P$ value $<0.05$ was considered statistically significant.

\section{Overall knowledge and attitudes about AS}

Four questions assessed knowledge and attitudes toward the term "antimicrobial stewardship." Two questions assessed the nurses' general knowledge by asking respondents to rate how familiar they were with the term, using a 4-item scale (not at all familiar to very familiar) and their knowledge of AS on a 0-10 Likert-type scale ( $0=$ no knowledge; 10 = expert knowledge). Respondents were also asked to rank how important they thought an AS program was in their health care setting using a 5-item scale (not at all important to extremely important). The overall knowledge scale was normalized to a 5-point scale for analysis purposes.

\section{Delivery of antimicrobials}

The questionnaire included 6 questions regarding nurses' delivery of antimicrobials: (1) the percentage of time that they were aware of the antimicrobial indication (0\%-100\%); (2) who they typically asked when they had questions about antimicrobial delivery (charge nurse, physician, pharmacist, etc); (3) if they had questioned a provider about an antimicrobial order in the last 30 days (yes/no); (4) if they were comfortable raising concerns about antimicrobial use (yes/no); (5) if they had given antimicrobials they thought were inappropriate (yes/no); and (6) whether they thought it was the nurses' role to improve antimicrobial use (yes/no).

\section{Knowledge and attitudes about antimicrobial use}

Eight statements regarding antimicrobial use were included, which asked participants to indicate their agreement on a 1-5 Likerttype scale ( 1 = strongly disagree; 5 = strongly agree; $0=$ do not know). Examples included: "The use of antimicrobials can cause harm," and "It is my responsibility to ensure appropriate antimicrobial use in my health care setting."

\section{Knowledge and attitudes about antimicrobial resistance}

Nurses were asked to rank their agreement with 9 statements about antimicrobial resistance on a 1-5 Likert-type scale ( 1 = strongly disagree; 5 =strongly agree; $0=$ don't know). Examples included: "Antimicrobial resistance is a significant problem nationally" and "Inappropriate use of antimicrobials causes resistance." The nurses were also given a list of possible interventions (online modules, chart audits, antimicrobial restrictions, etc) and asked to identify which interventions they believed would be most helpful to improve appropriate use of antimicrobials in their facility.

\section{Resources and education about antimicrobials}

Nurses were asked, "What interventions do you think would be most helpful in improving the appropriate use of antibiotics in your facility?" They were asked to check all that apply and were given 7 choices and 1 open-ended response option.

\section{RESULTS}

A total of 343 complete survey responses were received. Twentyseven were excluded because the nurse's primary role was not at the bedside. The final sample included 316 bedside nurses from 3 hospitals (15.8\% response rate). The sample was mostly women $(n=283$; $89 \%)$ and white $(n=296 ; 93 \%)$ with $64 \%$ having a baccalaureate degree or higher $(n=199)$. The respondents ranged in age from 2170 years (mean $=39$ years; $S D=11.9$ ). They reported $0-40$ years of experience $($ mean $=11.2$ years; $S D=10$ years $)($ Table 1$)$.

\section{Overall knowledge and attitudes about AS}

Fifty-two percent of staff nurses $(n=164)$ were not at all familiar with the term "antimicrobial stewardship," and $27 \%(n=85)$ reported having heard of the term but did not understand what it meant. The mean overall self-reported knowledge of AS was $1.54(\mathrm{SD}=1.2)$ on a 0 to 5-point Likert-type scale. Regarding the importance of having an AS program in their health care setting, 39.6\% $(n=125)$ of nurses indicated that it was moderately or extremely important, whereas $10 \%$ $(n=31)$ indicated it was not important or they did not know 
Table 1

Survey demographics

\begin{tabular}{|c|c|c|c|c|c|}
\hline Health Care Setting & $\begin{array}{l}\text { Diploma/Associates } \\
\mathrm{n}(\%)\end{array}$ & $\begin{array}{l}\text { Baccalaureate or Higher } \\
\mathrm{n}(\%)\end{array}$ & Women n (\%) & $\begin{array}{l}\text { White } \\
\mathrm{n}(\%)\end{array}$ & $\begin{array}{l}\text { Total } \\
\mathrm{N}(100 \%)\end{array}$ \\
\hline 40 beds & $6(37.5 \%)$ & $10(62.5 \%)$ & $15(93.8 \%)$ & $14(87.5 \%)$ & 16 \\
\hline 250 beds & $22(29.3 \%)$ & $53(70.7 \%)$ & 67 (89.3\%) & $72(96 \%)$ & 75 \\
\hline 500 beds & $81(38.2 \%)$ & $131(61.8 \%)$ & $189(89.2 \%)$ & 197 (92.95\%) & 212 \\
\hline Total & $109(36 \%)$ & $199(64 \%)$ & $283(89.4 \%)$ & 296 (93.4\%) & 303 \\
\hline
\end{tabular}

Thirteen respondents did not provide their demographic information.

(mean = 3.6; $\mathrm{SD}=1.6$ ). There was no statistical difference in familiarity, overall AS knowledge, and importance of having an AS program by location, education level, or ethnicity $(P>.05)$. There was also no relationship between AS knowledge, age, and years of experience $(r=0.10$ and $0.067 ; P>.05)$. There was a positive relationship between the familiarity of AS $(r=0.568 ; P=.000)$, and importance of having an AS program $(r=0.519 ; P=.000)$ with overall knowledge of AS. This means that those who reported a high knowledge of AS were both familiar with the term and believed it was important to have an AS program in their setting.

\section{Delivery of antimicrobials}

When giving an antimicrobial, 87\% $(\mathrm{n}=274)$ of the nurses reported knowing the reason their patient received it (Fig 1). There was no statistical difference in response to knowing the reason for an antimicrobial order based on age, degree, sex, or ethnicity. When asked, $15 \%-25 \%(n=49-83)$ of nurses reported they questioned the antimicrobial choice, dose, route, or duration within the past 30 days. Nurses with a baccalaureate degree or higher were significantly more likely to question antimicrobial choice, dose, and duration of antimicrobials compared with nurses with an associate degree or diploma, $X^{2}(1,307)=5.19 ; P=.023$ (Fig 1$)$. Thirty percent of nurses with a baccalaureate degree or higher reported having given an antimicrobial they thought was inappropriate, compared with $17 \%$ of nurses with an associates/diploma degree, $X^{2}(1,265)=8.32 ; P=.004$ (Fig 1$)$.
Nurses were asked to identify all the persons they ask when they had questions about antimicrobials (check all that apply). Nurses most commonly indicated they asked the primary medical team $(n=208 ; 66 \%)$, the charge nurse $(n=138 ; 43 \%)$, other nurses $(n=116$; $36 \%)$, or they asked the pharmacist $(n=111 ; 35 \%)$ for clarification. Almost all nurses (95\%) believed that they should be involved in interventions to improve antimicrobial use, and $86 \%$ were comfortable raising concerns.

\section{Knowledge and attitudes about antimicrobial use}

For questions related to antimicrobial use, the highest scores (1-5 scale) were "Strong knowledge about antimicrobials is important in my job" ( mean $=4.2 ; \mathrm{SD}=0.93$ ) and "I would like more education about antimicrobial use" (mean $=4.0 ; \mathrm{SD}=1.0$ ) (Fig 2). The lowest scores (1-5 scale) were "Antimicrobials are overused in my health care setting" ( mean $=2.3 ; \mathrm{SD}=1.4$ ) and "Appropriate use of antimicrobials can cause antimicrobial resistance" ( mean $=3.0 ; \mathrm{SD}=1.6$ ). There was no difference in the mean responses based on hospital, sex, or ethnicity. There was a difference in 3 of the 8 questions by education level. For example, nurses with a baccalaureate degree or higher agreed more strongly that "antimicrobial knowledge was important to their job," $F(1,340)=3.9 ; P=.033$, that they would "like more education on the appropriate use of antimicrobials," $F(1,339)=$ $4.0 ; P=.046$ and that "antimicrobials are over used in my health care

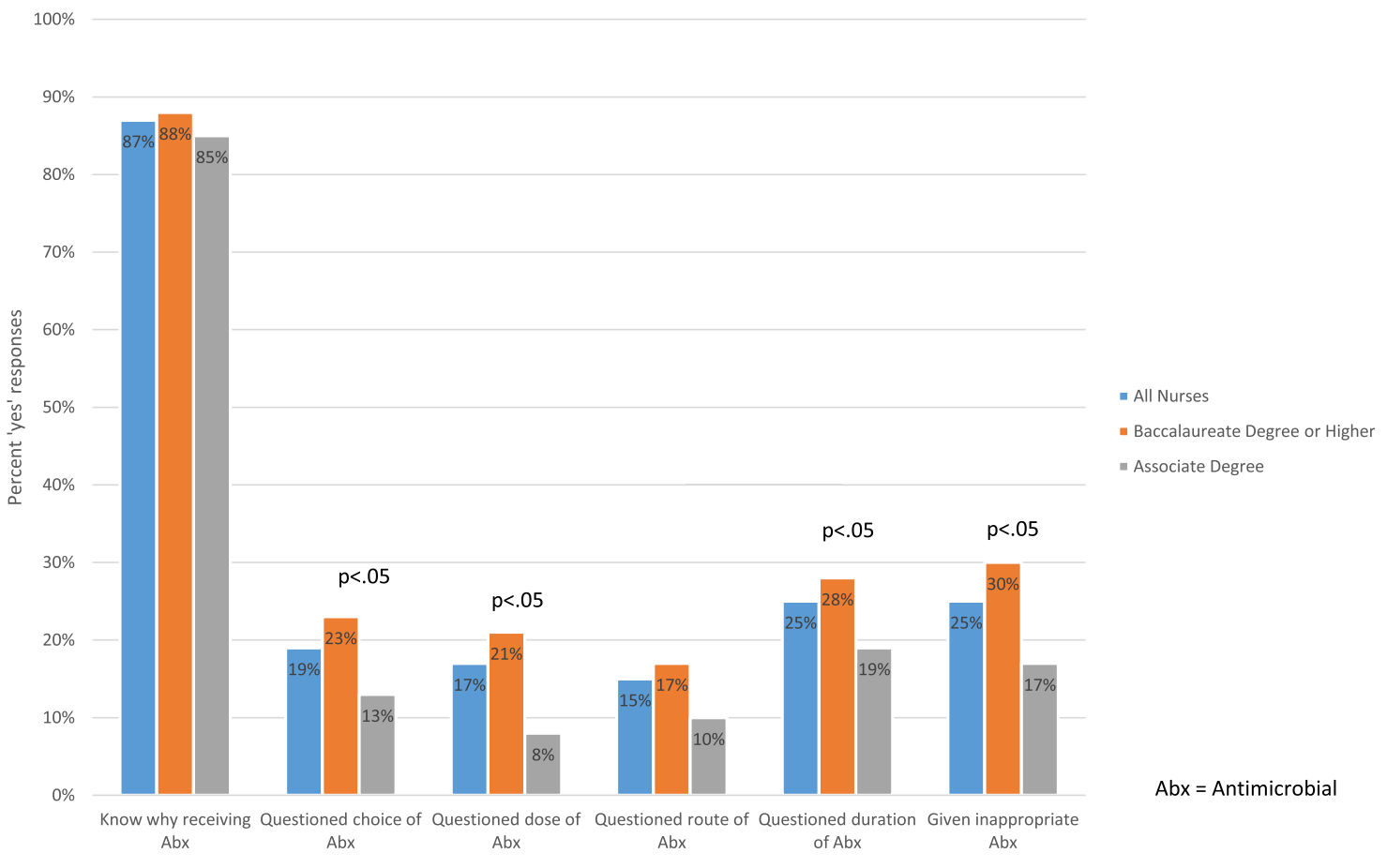

Fig 1. Delivery of antimicrobials for all nurses and by education level. Percent 'yes' responses. 


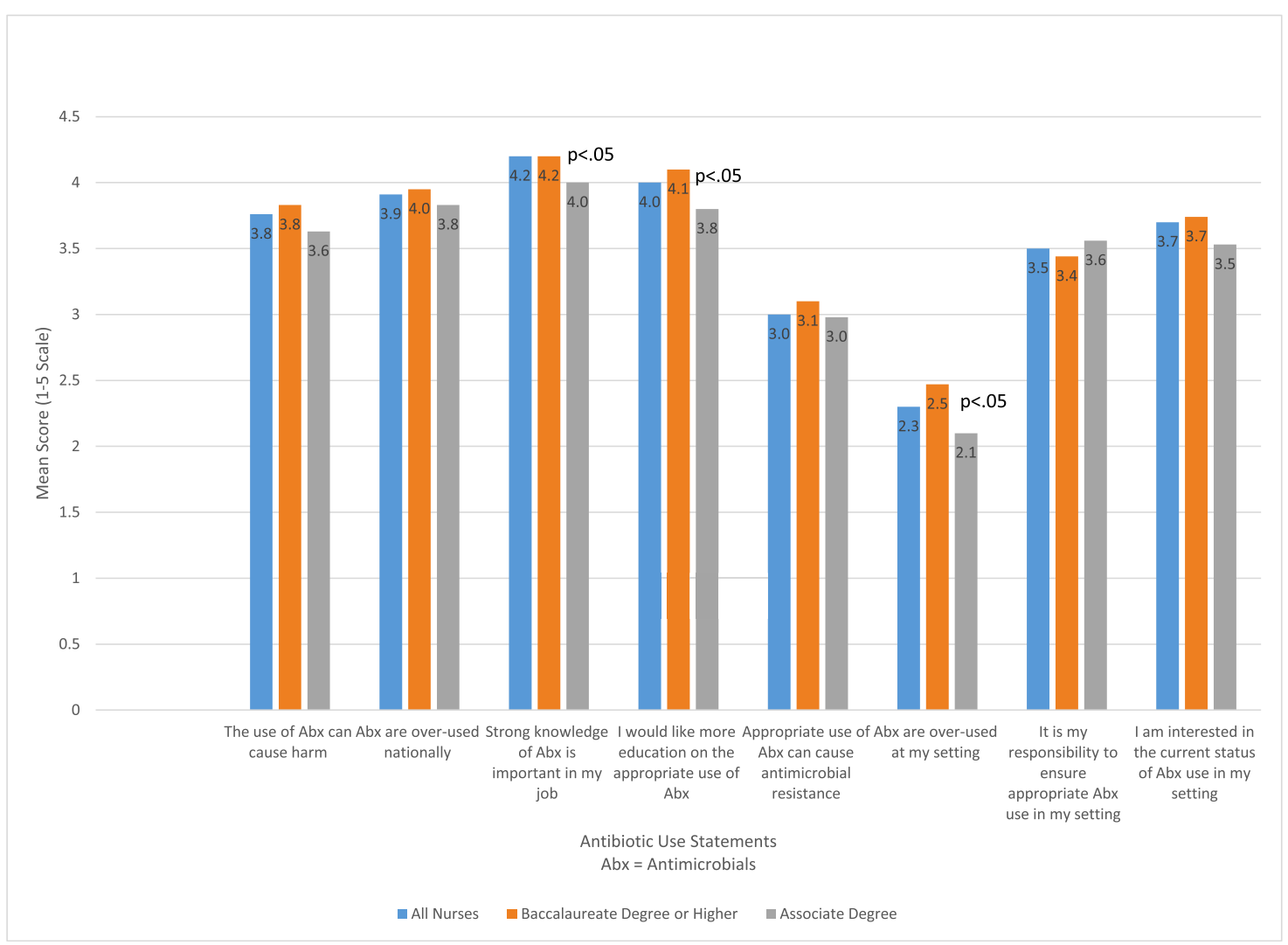

Fig 2. Knowledge and attitudes about antimicrobial use for all nurses and by education level.

setting" $F(1,340)=5.9 ; P=.016$ than nurses with a diploma/associates degree did (Fig 3).

There was a very weak negative correlation between years of experience and "I would like more education on the appropriate use of antimicrobials" ( $r=-0.199)$, and years of experience and "antimicrobials are overused at my health care setting" ( $r=-0.153)$. Cronbach's alpha for the 8 items addressing knowledge and attitudes about antibiotic use was 0.677

\section{Knowledge and attitudes about antimicrobial resistance}

For questions related to antimicrobial resistance, the highest scores (1-5 scale) were "Inappropriate use of antimicrobials causes resistance" (mean =4.6; $\mathrm{SD}=0.78$ ) and "Assessment and documentation of allergies are important to ensure appropriate antimicrobial use" (mean $=4.5 ; \mathrm{SD}=0.87$ ) (Fig 3). The lowest scores (1-5 score) were "Appropriate use of antimicrobials can cause resistance" (mean $=2.4$; $\mathrm{SD}=1.4$ ) and "Antimicrobial resistance is a significant problem in my health care setting" (mean $=2.7 ; \mathrm{SD}=1.7$ ). There was no difference by hospital, sex, or ethnicity and no significant correlations with age or years of experience. However, there was a difference in 1 of the resistance questions by education level. Nurses with a baccalaureate degree or higher agreed more strongly that "Prescribing broad-spectrum antimicrobials when equally effective narrower spectrum antimicrobials are available increases resistance" compared with nurses with an associates/diploma (Fig 3). The Cronbach's alpha for the 10 items addressing knowledge and attitudes about antimicrobial resistance was 0.724.

\section{Resources and education about antimicrobials}

Nurses were asked, "What interventions do you think would be most helpful in improving the appropriate use of antibiotics in your facility?" Respondents had the option to check all that apply. A majority said that evaluating antimicrobials after 48 hours of therapy would be a helpful intervention (74\%) and that pharmacists should be involved in AS-related interventions (68\%). Nurses also recommended online learning modules (46\%), knowledge of accurate allergy reporting (41\%), antimicrobial restrictions (39\%), auditing of charts (29\%), and lectures about antimicrobials (23\%). Other open-ended responses included: ongoing continuing education, use of protocols, patient education, and better collaboration with physicians.

\section{DISCUSSION}

Nurses at the point of care are uniquely positioned to engage in AS activities. Although nurses in our study believed AS programs were important, and were eager to be involved, many were not aware of AS nor did they understand their role. These findings underscore the need for IP education that focuses on increasing staff nurse awareness and demonstrating how nurses can make specific AS interventions.

Recent studies suggested that nurses were confident in assessing adverse drug reactions, obtaining cultures before antimicrobial delivery, and providing patient education. ${ }^{9}$ However, nurses were less confident in reviewing laboratory results and providing feedback about the appropriateness of prescribed antimicrobials. ${ }^{9}$ Specific strategies such as scripting for RNs, allergy verification, and online learning have been suggested to increase RN knowledge about AS. ${ }^{10-12}$ With appropriate training, nurses at the point of care are well positioned to identify opportunities for AS and to communicate these as part of the multidisciplinary team approach.

Successful AS strategies are best executed by interdisciplinary teams. ${ }^{2,4,13}$ For teams to be successful, each member must understand their role and the role of others on the team. In our study, 


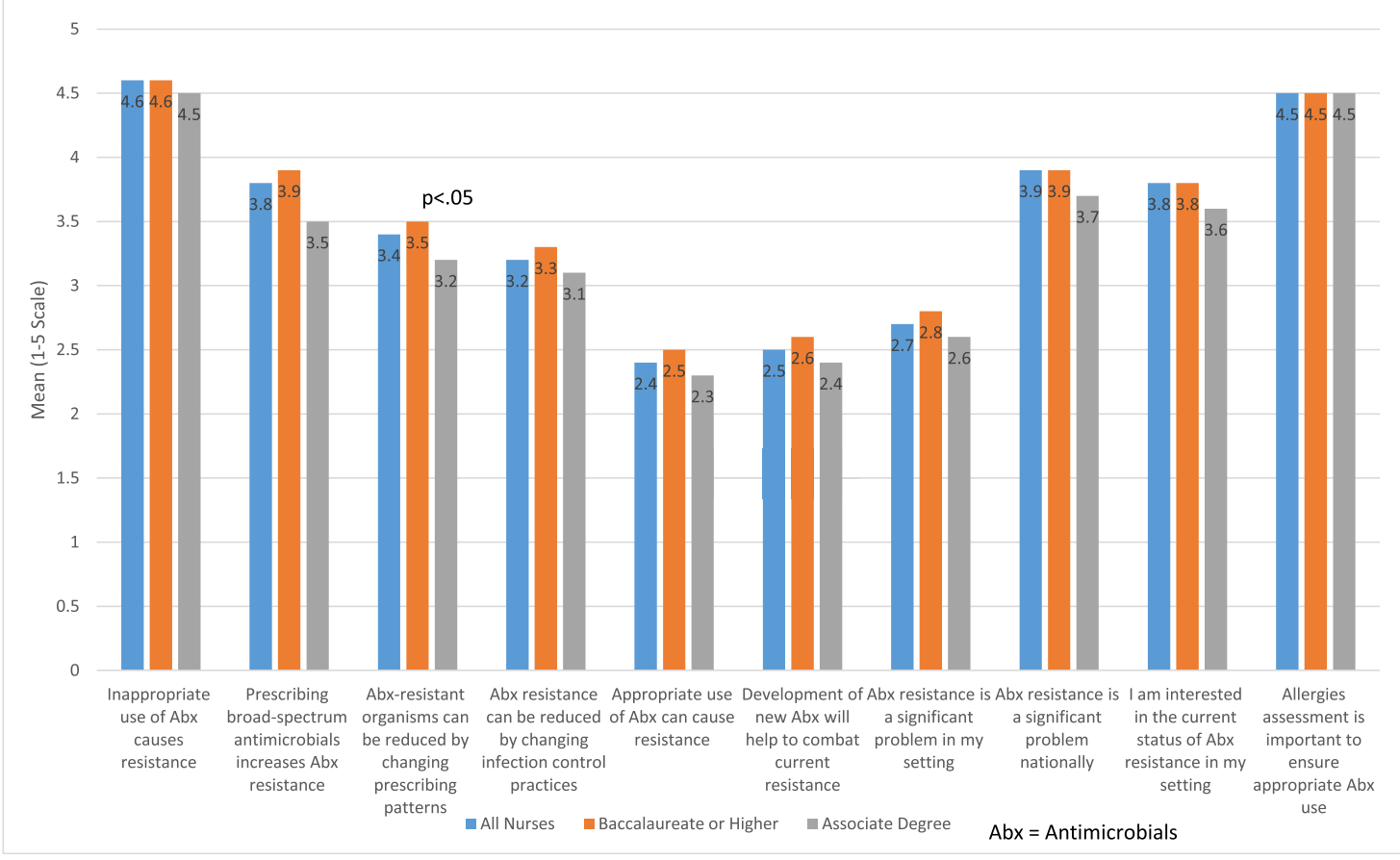

Fig 3. Knowledge and attitudes about antimicrobial resistance by all nurses and education level.

many nurses were not familiar with the term "antimicrobial stewardship." Most nurses believed they should be involved in AS; however, when given a choice of interventions to support AS, many believed pharmacist interventions would be most helpful to improve appropriate use of antimicrobials in their facility. This type of response underscores the opportunity to increase awareness of AS among nurses and to educate them to leverage their unique roles to advance AS. It is unrealistic to expect nurses to be full partners in a program they do not understand or value. It was encouraging that most nurses in our study believed they should be involved in interventions to improve antimicrobial use and they expressed a desire for additional education related to antimicrobials.

Despite the lack of familiarity with AS, most nurses in our study understood the global problem of antimicrobial resistance. However, most did not think antimicrobial resistance was a problem in their facility. One suggestion to address this discrepancy is to provide a facility-specific antibiogram to help nurses understand local antimicrobial resistance patterns. ${ }^{13}$ Sharing changes in resistance patterns with the AS team, including nurses at the point of care, can motivate staff to follow infection prevention protocols. ${ }^{14}$

Nurses in our study reported they generally knew why they were giving antimicrobials and were comfortable raising concerns; however, nurses also reported giving antimicrobials they thought were inappropriate. This discordance is concerning and has significant patient safety implications. It could be a result of lack of provider availability (eg, night shift) or lack of a "speak up" culture. ${ }^{15}$ Additional data are needed to define better and develop solutions to address this issue. Our survey also identified answer discrepancies based on degree type-nurses with baccalaureate degrees or higher reported more knowledge about broad-spectrum antimicrobials, that antimicrobials are more important in their job, and that they were more likely to question antimicrobial use than nurses with an associates/diploma were. These are interesting findings that need more exploration. One possible reason for these findings is that baccalaureate-nursing programs may include more pharmacology courses; however, this is not well reported in the literature. ${ }^{16,17}$ Regardless of nurses' variable knowledge of antimicrobials, these findings highlight a considerable opportunity for education targeting nurses.

Another interesting finding is that nurses in this study reported that accurate allergy identification would help improve the appropriate use of antimicrobials. This is important because previous research indicates that $90 \%$ of patients who report penicillin allergies may not indeed have an allergy. ${ }^{18}$ One common responsibility for nurses is to take an accurate allergy history. Our results suggest that nurses are both willing and interested in expanding this role to include exploring and documenting the nature of the allergy. ${ }^{17,19}$ Although nurses understand allergy reporting is essential, and admission questions about the presence of antimicrobial allergies are standard of care, they may not understand the need to verify true allergies, and there may not be processes in place to support more detailed allergy information gathering. ${ }^{18}$ One recent study suggested the use of scripting, structured allergy questionnaires, and patient education to elicit the true nature of antimicrobial allergies. ${ }^{10}$ Increased nursing focus on accurate allergy documentation represents a significant potential benefit to AS programs.

\section{Implications for IPS}

From these findings, we identified gaps in nursing knowledge regarding AS, but nurses also indicated interest in education and participation in AS activities. IPs have advanced training in antimicrobials, microbiology, and infection prevention strategies. Most recommendations include IPs in AS programs but fail to emphasize the critical role the IP plays in nursing education. ${ }^{3,5,7}$ IPs, based on their training, role, and presence in daily hospital activities, are well positioned to engage nurses and provide useful tools for AS. 
In this study, many nurses reported asking the medical team and pharmacist when they had questions about antimicrobials, but some reported asking the charge nurse. When implementing education, IPs may want to focus on charge nurses, as they are often the source of information for nurses at the point of care. This approach is supported by the findings of Amiri et $\mathrm{al}^{20}{ }^{20}$ who reported that empowering nurses and their supervisors resulted in an improvement in safety culture. Further, Monsees et $\mathrm{al}^{9}$ reported that interprofessional collaboration is essential to enhance nurse's participation in AS.

Nurses in our study acknowledged that they would like more education regarding antimicrobial use. In a 2016 commentary article, Ruis et $\mathrm{al}^{21}$ proposed that educational interventions need to supersede lectures, classes, or video presentations and be more focused on systems perspective education. They suggest a cognitive simulation approach to teach complex issues related to health care-acquired infections. ${ }^{21}$ These types of innovative approaches will be needed as we strive to educate today's nurses. Whatever educational approaches are used, IPs should also remember to include them in new hire orientation.

\section{Limitations}

There were some limitations to this study. This descriptive study included only inpatient nurses who worked at 3 hospitals within the same health care system, so the findings may not be generalizable to other inpatient facilities or ambulatory settings. The hospitals had recently implemented AS teams that included physicians, pharmacists, and IPs. However, they had yet to include point-of-care nurses nor had they implemented any point-of-care interventions. The survey was modified from an instrument used with physicians and other prescribers; therefore, despite the validation process, the questions may not have been optimized for application to nursing practice. Face validity was obtained from AS stewards and IPs and did not include staff nurses. Questions were on different scales (0-10 and 0-5) requiring standardization of the results. The overall response rate was low, but it was still within ranges reported by other investigators conducting online surveys. ${ }^{22} \mathrm{~A}$ low response rate may represent non-respondent bias. To increase survey response, researchers may consider decreasing the number of survey items. ${ }^{23}$

\section{Future research and education}

Our study identified a need for nurse education and engagement in AS-related efforts. Future research should address the effect of education on the knowledge and attitudes of staff nurses regarding AS and on nurse participation in AS interventions. Further work needs to explore how the interdisciplinary team communicates regarding antimicrobial use and how to empower nurses to speak up when they feel an antimicrobial may be inappropriate. Studies are also needed to understand better and leverage the role of staff nurses in AS programs, particularly in other health care settings such as officebased practices. Survey instruments regarding knowledge and attitudes of AS should be validated further in the staff nurse population.

\section{CONCLUSIONS}

Nurses at the point of care are uniquely positioned to collaborate with the interdisciplinary team on AS practices. This study identified that nurses in this particular health care system did not fully understand the purpose of the importance of AS nor did they recognize the many opportunities to contribute to AS endeavors. Based on their essential role in AS programs, IPs have an opportunity to expand education efforts with a focus on staff nurse knowledge and attitudes regarding AS. More research is needed on the impact of such an educational program. Finally, additional research into the dynamics of multidisciplinary AS collaboration between nurses, IPs, AS physicians, pharmacists, and front-line providers is needed.

\section{SUPPLEMENTARY MATERIALS}

Supplementary material associated with this article can be found in the online version at https://doi.org/10.1016/j.ajic.2019.03.022.

\section{References}

1. Centers for Disease Control and Prevention. 2017. Antimicrobial/antimicrobial resistance. Available from: https://www.cdc.gov/drugresistance/. Accessed May 13, 2019.

2. American Nurses Association \& Centers for Disease Control. 2017. Redefining the antimicrobial stewardship team: recommendations from the American Nurses Association/Centers for Disease Control and Prevention workgroup on the role of the registered nurses in hospital antimicrobial stewardship practices. Available from: https://www.cdc.gov/antimicrobial-use/healthcare/pdfs/ANA-CDC-whitepaper.pdf. Accessed May 13, 2019.

3. Moody J, Cosgrove SE, Olmsted R, Septimus E, Aureden K, Oriola S, et al. Antimicrobial stewardship: a collaborative partnership between infection preventionists and healthcare epidemiologists. Infect Control Hosp Epidemiol 2012;33:328-30.

4. Joint Commission. Antimicrobial stewardship. 2017. Available from: https://www. jointcommission.org/topics/hai_antimicrobial_stewardship.aspx. Accessed May 13, 2019.

5. Manning ML, Pfeiffer J, Larson EL. Combating antimicrobial resistance: the role of nursing in antimicrobial stewardship. Am J Infect Control 2016;44:1454-7.

6. Olans RN, Olans RD, DeMaria A. The critical role of the staff nurse in antimicrobial stewardship: recognized but already there. Clin Infect Dis 2016;62:84-9.

7. Manning ML, Giannuzzi D. Keeping patients safe: antimicrobial resistance and the role of nurse executives in antimicrobial stewardship. J Nurs Adm 2015;45:67-9.

8. Monsees E, Goldman J, Popejoy L. Staff nurses as antimicrobial stewards: an integrative literature review. Am J Infect Control 2017;45:917-22.

9. Monsees E, Popejoy L, Jackson MA, Lee B, Godman J. Integrating staff nurses in antimicrobial stewardship: opportunities and barriers. Am J Infect Control 2018;46:737-42.

10. Sumner S, Forsyth S, Collette-Merrill K, Taylor C, Vento T, Veillette J, et al. Antimicrobial stewardship: the role of clinical nurses and nurse educators. Nursing Educ Today 2018;60:157-60.

11. Rocha-Pereira N, Lafferty N, Nathwani D. Educating healthcare professionals in antimicrobial stewardship: can online-learning solutions help? J Antimicrob Chemother 2015;70:3175-7.

12. Wentzel J, van Drie-Perik R, Nijdam L, Geesing J, Sanderman R, van Gemert-Pijnen JE. Antimicrobial information application offers nurses quick support. Am J Infect Control 2016;44:677-84.

13. Patterson JE, Malani PN, Maragakis LL. Infection control in the intensive care unit: progress and challenges in systems and accountability. Crit Care Med 2010;38 (8 Suppl):265-8.

14. McClung L, Obasi C, Knoblock M, Safdar N. Health care worker perspectives of their motivation to reduce health care-associated infections. Am J Infect Control 2017:45:1064-8

15. Alingh CW, van Wijngaarden JDH, van de Voorder K, Paauwe J, Huijsman R. Speaking up about patient safety concerns: the influence of safety management approaches and climate on nurses' willingness to speak up. BMJ Qual Saf 2019;28:39-48

16. Bullock S, Manias E. The educational preparation of undergraduate nursing students in pharmacology: a survey of lecturers' perceptions and experiences. J Adv Nurs 2002;40:7-16.

17. Carter EJ, Greendyke WG, Furuya EY, Srinivasan A, Shelley AN, Bothra A, et al. Exploring the nurses' role in antimicrobial stewardship: a multisite qualitative study of nurses and infection preventionists. Am J Infect Control 2018;46:492-7.

18. Solensky R. Hypersensitivity reactions to beta-lactam antimicrobials. Clin Rev Allergy Immunol 2003;24:201-20.

19. Australian Society of Clinical Immunology and Allergy.2016. Sulfonamide antimicrobial allergy. Available from: https://www.allergy.org.au/images/pcc/ASCIA_PCC_Sulfonamide_antibiotic_allergy_2019.pdf. Accessed May 13, 2019.

20. Amiri M, Khademian Z, Nikandish R. The effect of nurse empowerment educational program on patient safety culture: a randomized controlled trial. BMC Med Educ 2018;18:158.

21. Ruis AR, Shaffer DW, Shirley DK, Safdar N. Teaching health care workers to adopt a systems perspective for improved control and prevention of health care-associated infections. Am J Infect Control 2016;44:1360-4.

22. Bergeson SC, Gray J, Ehrmantraut LA, Laibson T, Hays RD. Comparing web-based with mail survey administration of the Consumer Assessment of Healthcare Providers and Systems (CAHPS) clinician and group survey. Primary Health Care 2013;3:132.

23. Turk T, Elhady MT, Rashed S, Abdelkhalek M, Nasef SA, Khallaf AM, et al. Quality of reporting web-based and non-web-based survey studies: what authors, reviewers, and consumers should consider. PLoS One 2018;13:e0194239. 